Einführung in das Schwerpunktthema: Sustainable Corporate Governance

\title{
Neuer Ansatzpunkt für nachhaltige Unternehmensführung?
}

$\sigma^{0}$

Von Kathrin Ankele und Michael Keil orporate Governance oder gute Unternehmensführung bezeichnet üblicherweise die Verantwortlichkeit des Managements gegenüber den Shareholdern. Diese Auslegung wird angesichts veränderter Machtverhältnisse zwischen Unternehmen und Staat sowie des Leitbildes der Nachhaltigen Entwicklung als zu eng erachtet. Unternehmen sind mit der Forderung konfrontiert, gesellschaftliche Verantwortung zu übernehmen und gute Unternehmensfiuhrung auf soziale und ökologische Erfordernisse auszuweiten.

Darüber hinaus haben die Unternehmensskandale der letzten Jahre Zweifel an der Wirksamkeit bestehender Regelungen zur Unternehmensführung aufkommen lassen. Um diesem Vertrauensverlust zu begegnen, erstellte eine Regierungskommission letztes Jahr den Deutschen Corporate Governance Kodex (DCGK). Dieser will ,das Vertrauen der internationalen und nationalen Anleger, der Kunden, der Mitarbeiter und der Öffentlichkeit in die Leitung und Überwachung deutscher börsennotierter Aktiengesellschaften fördern“. In Abschnitt 4.1.1 wird der Vorstand auf das Unternehmensinteresse und die Steigerung des nachhaltigen Unternehmenswertes verpflichtet (1).

Inwiefern sich darin ein belastbarer und ausbaufähiger Verweis auf Corporate Sustainability ausdrückt ist umstritten, wie auch in den Beiträgen dieses Schwerpunktthemas von Josef Wieland, Axel Klein und Andreas Steinert deutlich wird. Unter dem Stichwort „Sustainable Corporate Governance" möchten wir eine Diskussion darüber anstoßen, ob in der Schnittmenge aus ,,aufgeklärter" Corporate Governance und Instrumenten der Corporate Sustainability neue Ansatzpunkte für eine den Prinzipien der Nachhaltigkeit verpflichtete Unternehmensführung entwickelt werden können.

Voraussetzung hierfür ist zum einen, die Selbststeuerungsfähigkeit von Unternehmen in Richtung Nachhaltigkeit weiter zu entwickeln. Zum anderen sind auch die Zivilgesellschaft insgesamt und insbesondere NGOs, Interessenverbände und wissenschaftliche Einrichtungen gefordert, Problemdruck sowie Nachfrage nach nachhaltiger Unternehmensführung zu erzeugen. Auch die Medien spielen eine wichtige Rolle.

\section{- Instrumente unternehmerischer Selbststeverung}

In den letzten Jahren wurden verschiedene Konzepte und Instrumente der nachhaltigen Unternehmensführung entwickelt. Dazu zählen neben Corporate Sustainability auch Corporate Social Responsibility (CSR) oder Corporate Citizenship (CC). Während Corporate Sustainability erst seit dem Umweltgipfel 1992 in Rio de Janeiro verstärkt diskutiert wird, kann CSR im anglo-amerikanischen Raum auf eine Entwicklungsgeschichte bis in die 1950er Jahre zurückblicken(2). Corporate Sustainability zielt auf die Umsetzung der drei Dimensionen der Nachhaltigkeit, Ökologie, Ökonomie und Soziales. In der Praxis war zunächst überwiegend eine Fortsetzung des betrieblichen Umweltschutzes in neuem Gewand zu beobachten. In letzter Zeit dominiert zunehmend die ökonomische Nachhaltigkeit.

In der Entwicklungsgeschichte von CSR wurden verschiedene Vorstellungen von ,,sozialer Verantwortung" entwickelt. Sie haben gemeinsam, dass

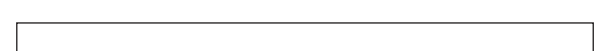

Einen Überblick über alle bisher erschienenen Hefte des Informationsdienstes "Ökologisches Wirtschaften" finden Sie auf unseren Internetseiten unter der Adresse:

www.oekom.de

Wir freuen uns auf Ihren Besuch!
Engagement über ökonomische Ziele und gesetzliche Verpflichtungen hinaus erwartet wird, das je nach Ansatz bis zu einer aktiven Verbesserung der sozialen Umwelt unter Berücksichtigung sich ändernder gesellschaftlicher Werte und Prioritäten reichen kann.

In Europa hat CSR vor allem durch das „Green Paper" der EU-Kommission und das nachfolgend einberufene Multistakeholderforum, das Vorschläge zur Operationalisierung erarbeiten soll, an Bedeutung gewonnen (3).

Seit einiger Zeit ist eine Annäherung dieser beiden Ansätze zu beobachten. Kritisch betrachtet geht damit eine Aufweichung der ökologischen Dimension zugunsten der ökonomischen und einzelnen Aspekten der sozialen Dimension einher. Positiv zu bewerten ist eine stärkere Verankerung auf der strategischen Ebene in Unternehmen. Die Erfahrungen mit Umweltmanagement haben gezeigt, dass eine ausschließlich operative Herangehensweise mittelfristig und in ökonomischen Krisenzeiten zu Bedeutungsverlust führen kann.

\section{Stärken und Schwächen der Instrumente}

Diese freiwilligen Instrumente der betrieblichen Selbststeuerung weisen sowohl Stärken als auch Schwächen auf. Zu den Stärken zählt, dass innerhalb der Unternehmen durch die Zielorientierung und die möglichst breite Verankerung in der Organisation Innovationen und integrierte Maßnahmen gefördert werden. Ferner kann bei einem offenen Umgang mit Informationen und einer aktiven Kommunikation mit Anspruchsgruppen das Vertrauen zwischen Unternehmen einerseits sowie Behörden und der Öffentlichkeit andererseits gesteigert werden. Nicht zuletzt gibt es etliche Beispiele dafür, dass freiwillige Instrumente Probleme besser lösen können als klassische Regulierung, wenn die Lösungen durch den Markt honoriert werden.

Daneben weisen freiwillige Ansätze jedoch auch eine Reihe von Schwächen auf: So sind sie ungeeignet für Bereiche, in denen kein unternehmerisches Eigeninteresse besteht, wenn also weder Kostensenkungen noch Imageverbesserungen erreicht oder strengere gesetzliche Regelungen vermieden werden können. Daneben besteht die Gefahr des green- und bluewashing. Darunter ist zu verstehen, dass Unternehmen öffentliche Anerkennung durch die Teilnahme an Umwelt- oder UN-Programmen wie dem Global Compact erhalten, ohne die entsprechenden Anforderungen einzulösen. 


\section{- Erfolgsbedingungen}

Ob die Erwartungen erfüllt werden, die in diese Instrumente gesetzt werden, ist von verschiedenen unternehmensinternen und -externen Bedingungen abhängig.

Zunächst sind Unternehmen aufgefordert, gesellschaftliche Anforderungen und Zielsetzungen zu berücksichtigen. Dies kann sowohl direkt durch die Einbeziehung von Stakeholdern in die Festlegung von Zielen und Programmen wie beispielsweise in Multistakeholderansätzen geschehen. Oder aber durch aktives Issue-Management unter expliziter Berücksichtigung gesamtgesellschaftlicher Ziele, die in einer nationalen Nachhaltigkeitsstrategie vorgegeben oder einem Umweltplan festgehalten werden. Bislang tun sich Unternehmen allerdings schwer, Stakeholder aktiv einzubeziehen. Hierfür müssen spezifische Kapazitäten wie Dialogfähigkeit und Frühwarnsysteme erst noch aufgebaut werden.

Doch auch der Zivilgesellschaft kommt eine wichtige Rolle zu. Sie muss eine kontinuierliche Hintergrundkontrolle der Unternehmensaktivitäten sicherstellen und dabei sowohl über Honorierungs- als auch über Sanktionsmechanismen verfügen. Sinnvollerweise sind hierfür unabhängige Experten wie NGOs, Verbände oder wissenschaftliche Einrichtungen erforderlich, die diese Aufgaben im Interesse und in Vertretung der Zivilgesellschaft wahrnehmen. Eine zentrale Voraussetzung hierfür ist allerdings, dass diese Experten über die notwendigen Ressourcen und Kapazitäten verfügen. Davon ist derzeit noch nicht auszugehen, was auch im Beitrag von Jens Martens deutlich wird.

Darüber hinaus existieren Bereiche, deren Schutz weder über das Eigeninteresse der Wirtschaft noch über eine mobilisierte Öffentlichkeit sichergestellt werden kann. Hierfür ist es notwendig, dass der Staat Regulierungskompetenzen aufrecht erhält oder stärkt.

„Sustainable Corporate Governance“ erfordert somit Entwicklungen in Bezug auf die Selbststeuerungsfähigkeit der Unternehmen und in Bezug auf die Steuerungskapazitäten der Zivilgesellschaft und des Staates.

\section{- Die Beiträge im Überblick}

Die Beiträge dieses Schwerpunktes gehen mit unterschiedlichen Perspektiven und Schwerpunktsetzungen auf diese Steuerungskompetenzen ein.
Josef Wieland beschreibt, wie Unternehmen durch die Implementierung eines Wertemanagementsystems eine wesentliche Grundlage für eine verantwortungsvolle Unternehmensführung und wirksame Corporate-Governance-Programme legen können.

Dass die Nachhaltigkeitsprogramme vieler Unternehmen bislang jedoch oft an den Interessen und Anforderungen ihrer Stakeholder vorbeigehen, zeigen Axel Klein und Andreas Steinert in „Bitte nachbessern“. Sie stellen die Ergebnisse einer Internetbefragung von Stakeholdern vor und folgern, dass Unternehmen im Rahmen ihrer Berichterstattung stärker auf ihre Stakeholder eingehen müssen.

Auf Grund der geringen öffentlichen Akzeptanz freiwilliger Selbstverpflichtungen setzen Unternehmen verstärkt darauf, Stakeholder direkt an der Erarbeitung von Nachhaltigkeitsprogrammen zu beteiligen. Peter Utting, beschreibt Vor- und Nachteile solcher Multistakeholder-Initiativen und unterbreitet Vorschläge zu deren Verbesserung.

Jens Martens diskutiert die Reichweite von Instrumenten der unternehmerischen Selbststeuerung aus der Perspektive von NGOs. Darüber hinaus beschreibt er eine Mehr-Ebenen-Strategie, die auf dem Weltsozialforum in Porto Alegre verabschiedet wurde und die künftige Positionierung von NGOs gegenüber Corporate Accountability enthält.

Neben NGOs stellen auch Anlageberater und Vermögensverwalter eine zunehmend wichtige An-

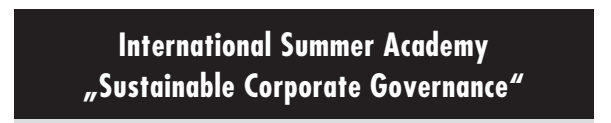

Deutschland, Sommer 2004

Wann gelingt der Übergang von "Corporate Governance", also der Verantwortlichkeit des Managements gegenüber den Shareholdern, zu "Sustainable Corporate Governance"? Ausschlaggebend sind vor allem zwei Faktoren: die Wirksamkeit zivilgesellschaftlicher Steuerung und die Fähigkeit der Wirtschaft, sich selbst in Richtung Nachhaltigkeit zu steuern. Auf der Summer Academy diskutieren internationale Expertinnen und Experten aus Wissenschaft und Praxis Chancen und Grenzen bestehender Ansätze der Unternehmenssteuerung sowie Weiterentwicklungsperspektiven. Die Summer Academy ist Teil des IÖW-Forschungsprojekts "Governance and Sustainability", in dem das IÖW den Reformdruck auf herkömmliche Regelungssysteme sowie Chancen für eine nachhaltige Gesellschaftsentwicklung untersucht.

Kontakt: kathrin.ankele@ioew.de,

Informationen: www.ioew.de/governance. spruchsgruppe für Unternehmen dar. Thomas Loew untersucht in seinem Beitrag, inwieweit unterschiedliche ökologisch-ethische Ratingverfahren Einfluss auf Unternehmensstrategien ausüben.

Vor dem Hintergrund der eher verhaltenen Zwischenbilanz zur Wirksamkeit unternehmerischer Selbststeuerung in Richtung Nachhaltigkeit stellt sich abschließend die Frage, welche Rolle der Staat in diesem Zusammenhang spielen kann und sollte. Christian Hey argumentiert, dass vor dem Hintergrund der Grenzen unternehmerischer Selbststeuerung dem Staat nach wie vor eine zentrale Rolle zukommt.

Auch wenn der politische Mainstream derzeit in Richtung Deregulierung und Abbau des öffentlichen Sektors zielt, zeigt die Diskussion um Sustainable Corporate Governance, dass die Leistungsfähigkeit des Staates und der Zivilgesellschaft zentral sind, um die Übernahme gesellschaftlicher Verantwortung durch Unternehmen sicherzustellen. Die hier begonnene Debatte um Sustainable Corporate Governance werden wir im Rahmen einer Veranstaltung fortsetzen (siehe Kasten).

\section{Anmerkungen}

(1) Regierungskommission Deutscher Corporate Governance Kodex unter www.corporate-governance-code.de.

(2) vgl. Caroll, A.: Corporate Social Responsibility. Evolution of a Definitional Construct. In: Business \& Society, 38, 3, 1999.

(3) EU-Kommission: Promoting a European Framework for corporate social responsibility. Green paper, 2001. Im Internet unter http://europa.eu.int/comm/employment_ social/soc-dial/csr/greenpaper_en.pdf.

\section{Die AutorInnen}

Kathrin Ankele ist Leiterin, Michael Keil wissenschaftlicher Mitarbeiter im Forschungsfeld "Ökologische Unternehmenspolitik" am Institut für ökologische Wirtschaftsforschung (IÖW).

Kontakt: IÖW, Potsdamer Str. 105, 10785 Berlin, Tel. 030-884594-21, E-Mail: kathrin.ankele@ioew.de michael.keil@hannover.ioew.de 
(c) 20I0 Authors; licensee IÖW and oekom verlag. This is an article distributed under the terms of the Creative Commons Attribution Non-Commercial No Derivates License (http://creativecommons.org/licenses/by-nc-nd/3.o/), which permits unrestricted use, distribution, and reproduction in any medium, provided the original work is properly cited. 